\title{
Divisibility properties of Smith matrices
}

\author{
by
}

Shaofang Hong, Jianrong Zhao and Youzhan Yin (Chengdu)

1. Introduction. For any integers $x$ and $y$, we denote by $(x, y)$ (resp. $[x, y]$ ) the greatest common divisor (resp. least common multiple) of $x$ and $y$. Let $e \geq 1$ be an integer and $S=\left\{x_{1}, \ldots, x_{n}\right\}$ be a set of $n$ distinct positive integers. The $n \times n$ matrix

$$
\left(S^{e}\right)=\left(\left(x_{i}, x_{j}\right)^{e}\right),
$$

having the eth power $\left(x_{i}, x_{j}\right)^{e}$ as its $(i, j)$-entry, is called the eth power $G C D$ matrix on $S$. The $n \times n$ matrix

$$
\left[S^{e}\right]=\left(\left[x_{i}, x_{j}\right]^{e}\right),
$$

having the eth power $\left[x_{i}, x_{j}\right]^{e}$ as its $(i, j)$-entry, is called the eth power $L C M$ matrix on $S$. These are simply called the GCD matrix and LCM matrix respectively if $e=1$. The set $S$ is said to be factor closed (FC) if it contains every divisor of $x$ for any $x \in S$. The set $S$ is said to be $g c d$-closed if for all $i$ and $j,\left(x_{i}, x_{j}\right)$ is in $S$. Evidently, an FC set is gcd-closed but not conversely. A famous theorem of Smith [29] states that the determinant of the matrix $\left[(i, j)^{e}\right]$ equals $\prod_{k=1}^{n} J_{e}(k)$, where $J_{e}$ is the Jordan totient function (i.e. $J_{e}(x)=x^{e} \prod_{p \mid x}\left(1-1 / p^{e}\right)$ for any positive integer $x$ ). Smith also gave a formula for the determinant of the power LCM matrix $\left([i, j]^{e}\right)$. Since then many generalizations of Smith's results have been published; see, for example, $[1-4,7,8,12,14,19,27,28]$. Later on power GCD matrices and power LCM matrices are called Smith matrices. It is known that the power GCD matrix on any set is nonsingular, but an LCM matrix may be singular. There are some papers $([6,13,17-19,23,24])$ studying the nonsingularity of power LCM matrices; also, several authors (see [21, 22, $26,30]$ ) considered the eigenstructure of power GCD matrices and reciprocal power LCM matrices.

2000 Mathematics Subject Classification: Primary 11C20, 11A25.

Key words and phrases: gcd-closed set, lcm-closed set, greatest-type divisor, least-type multiple, Smith matrix, divisibility.

The research of Hong is supported by Program for New Century Excellent Talents in University Grant \# NCET-060785 and by SRF for ROCS, SEM. 
Divisibility is another central topic in the field of Smith matrices. Bourque and Ligh [5] showed that if $S$ is FC, then $\left(S^{e}\right) \mid\left[S^{e}\right]$ in the ring $M_{n}(\mathbb{Z})$ of $n \times n$ matrices over the integers. That is, there is an $M \in M_{n}(\mathbb{Z})$ such that $\left[S^{e}\right]=\left(S^{e}\right) M$, or equivalently, $\left(S^{e}\right)^{-1}\left[S^{e}\right] \in M_{n}(\mathbb{Z})$. Hong [16] proved that such factorization holds when $S$ is either a divisor chain or multiple closed (namely, $y \in S$ if $x|y| \operatorname{lcm}(S)$ for all $x \in S$, where $\operatorname{lcm}(S)$ means the least common multiple of all the elements of $S$ ). But such factorization is no longer true if $S$ is gcd-closed [15]. For $x, y \in S$ and $x<y$, if $x \mid y$ and the conditions $x|z| y$ and $z \in S$ imply that $z \in\{x, y\}$, then we say that $x$ is a greatest-type divisor of $y$ in $S$, and we also say that $y$ is a least-type multiple of $x$ in $S$. For $x \in S$, we denote by $G_{S}(x)$ and $L_{S}(x)$ the set of all greatest-type divisors of $x$ in $S$ and the set of all least-type multiples of $x$ in $S$ respectively. It follows from [15] that there is a gcd-closed set $S$ with $\max _{x \in S}\left\{\left|G_{S}(x)\right|\right\}=2$ such that $(S)^{-1}[S] \notin M_{n}(\mathbb{Z})$. However, it is not clear whether there is a gcd-closed set $S$ with $\max _{x \in S}\left\{\left|G_{S}(x)\right|\right\}=1$ such that $(S)^{-1}[S] \notin M_{n}(\mathbb{Z})$. Hong believed that the answer to this question should be negative. Actually, Hong [19] proposed the following conjectures.

Conjecture 1.1 ([19]). Let $S$ be ged-closed and $\max _{x \in S}\left\{\left|G_{S}(x)\right|\right\}=1$. Then the GCD matrix $\left(\left(x_{i}, x_{j}\right)\right)$ on $S$ divides the LCM matrix $\left(\left[x_{i}, x_{j}\right]\right)$ on $S$ in $M_{n}(\mathbb{Z})$.

Conjecture 1.2 ([19]). Let $S$ be lcm-closed and $\max _{x \in S}\left\{\left|L_{S}(x)\right|\right\}=1$. Then the GCD matrix $\left(\left(x_{i}, x_{j}\right)\right)$ on $S$ divides the LCM matrix $\left(\left[x_{i}, x_{j}\right]\right)$ on $S$ in $M_{n}(\mathbb{Z})$.

By [16] we know that Conjectures 1.1 and 1.2 are true when $S$ is a divisor chain. Feng, Tan and Zheng [10] showed that Conjecture 1.1 holds if $S$ consists of two relatively prime divisor chains. In this paper, we introduce a new method to investigate the above conjectures. We first show several theorems on the structure and properties of gcd-closed sets $S$ with $\max _{x \in S}\left\{\left|G_{S}(x)\right|\right\}=1$. Using these we then construct an integer matrix which equals the product $\left(S^{e}\right)^{-1}\left[S^{e}\right]$; see Theorem 2.5 below. This in particular implies Conjecture 1.1 is true. Next, we establish a result for the lcm-closed case which confirms Conjecture 1.2. Finally, we make some remarks on the finite arithmetic progression case and raise an open problem.

For any permutation $\sigma$ on $\{1, \ldots, n\}$, define $S_{\sigma}:=\left\{x_{\sigma(1)}, \ldots, x_{\sigma(n)}\right\}$. Then one can easily check that $\left(S^{e}\right)^{-1}\left[S^{e}\right]=P^{t}\left(S_{\sigma}^{e}\right)^{-1}\left[S_{\sigma}^{e}\right] P$, where $P$ is the $n \times n$ permutation matrix whose $i$ th row equals

$$
(0, \ldots, 0, \underbrace{1}_{\sigma(i)}, 0, \ldots, 0) \quad(1 \leq i \leq n) .
$$

It follows that $\left(S^{e}\right)^{-1}\left[S^{e}\right] \in M_{n}(\mathbb{Z}) \Leftrightarrow\left(S_{\sigma}^{e}\right)^{-1}\left[S_{\sigma}^{e}\right] \in M_{n}(\mathbb{Z})$. So for divisibility purposes, we can rearrange the elements of $S$ in case of necessity. 
Throughout the paper, for any finite sets $T$ and $Q$ of integers, we denote by $|T|$ and $\max (T)$ the cardinality of $T$ and the maximal element of $T$ respectively, and define $(T, Q):=(\max (T), \max (Q))$. Then $(x, T)=(x, \max (T))$ for any integer $x$.

2. The gcd-closed case. First we prove three results on the structure of certain gcd-closed sets.

Lemma 2.1. Let $n \geq 2$ and $x_{1}<\cdots<x_{n}$. If $\max _{x \in S}\left\{\left|G_{S}(x)\right|\right\}=1$ and $x_{i} \mid x_{n}$ for all $1 \leq i \leq n$, then $x_{1}|\cdots| x_{n-1} \mid x_{n}$.

Proof. We use induction on $n$. If $n=2$, then the result is obvious, so let $n \geq 3$.

Assume that the assertion is true for $n-1$. Now consider the case of $n$. Let $S^{\prime}=\left\{x_{1}, \ldots, x_{n-1}\right\}$. Then $x_{n-1} \mid x_{n}$ by assumption. Hence $x_{n-1}$ is a greatest-type divisor of $x_{n}$ in $S$.

We claim that $x_{j} \mid x_{n-1}$ for all $1 \leq j \leq n-2$. Indeed, otherwise there exists a $j, 1 \leq j \leq n-2$, such that $x_{j} \nmid x_{n-1}$. Let $J$ be the set of all such $j$ and put $j_{0}:=\max \{j: j \in J\}$. Then $x_{j_{0}}$ is another greatest-type divisor of $x_{n}$ in $S$. This means that $\left|G_{S}\left(x_{n}\right)\right| \geq 2$, a contradiction.

By the claim we know that $\max _{x \in S^{\prime}}\left\{\left|G_{S^{\prime}}(x)\right|\right\} \geq 1$. Since, on the other hand, $\max _{x \in S}\left\{\left|G_{S}(x)\right|\right\}=1$, we deduce that $\max _{x \in S^{\prime}}\left\{\left|G_{S^{\prime}}(x)\right|\right\}=1$. It follows from the claim and induction hypothesis that for the set $S^{\prime}$, we have $x_{1}|\cdots| x_{n-1}$. So $x_{1}|\cdots| x_{n}$ as required.

Let $S$ be gcd-closed and $\max _{x \in S}\left\{\left|G_{S}(x)\right|\right\}=1$. Then by Lemma 2.1, we can rearrange $S$ into "composite divisor chains" using the following iterative rule:

STEP 1. Pick the biggest element of $S$ and consider the set of all its divisors in $S$, denoted by $X_{1}=\left\{x_{11}, \ldots, x_{1, a_{1}}\right\}$, where $a_{1}=\left|X_{1}\right|$. By Lemma 2.1 , these numbers form a divisor chain.

SteP 2. If $X_{1}=S$, we are done. If $X_{1} \neq S$, then Step 1 applied to $S \backslash X_{1}$ gives us another divisor chain, denoted by $X_{2}=\left\{x_{21}, \ldots, x_{2, a_{2}}\right\}$, where $a_{2}=\left|X_{2}\right|$. If $X_{1} \cup X_{2}=S$, we are done. If $X_{1} \cup X_{2} \neq S$, then by Step 1 applied to $S \backslash\left(X_{1} \cup X_{2}\right)$, we get a new divisor chain $X_{3}$. Since $S$ is finite, by repeating Step 1 a finite number of times, we can classify $S$ into $k$ disjoint divisor chains $X_{1}, \ldots, X_{k}$, i.e., $S=\coprod_{1 \leq i \leq k} X_{i}$, where $X_{i}=\left\{x_{i 1}, \ldots, x_{i, a_{i}}\right\}$, $a_{i}=\left|X_{i}\right|, x_{i 1}<\cdots<x_{i, a_{i}}$.

Note that $x_{i 1}|\cdots| x_{i, a_{i}}$ for $1 \leq i \leq k$. Let $A:=\left\{a_{1}, a_{1}+a_{2}, \ldots, a_{1}+\cdots+\right.$ $\left.a_{k}\right\}$ and $a_{0}=0$. For $1 \leq i \leq k$ and $1 \leq j \leq a_{i}$, define $y_{a_{0}+a_{1}+\cdots+a_{i-1}+j}:=x_{i j}$. Now we rearrange the elements of $S$, and in Lemmas 2.2-2.4 and Theorem 
2.5(i) below, we always let

$$
S=\coprod_{1 \leq i \leq k} X_{i}=\left\{y_{1}, \ldots, y_{n}\right\}
$$

with $y_{1}=1$. Obviously $y_{a} \nmid y_{b}$ if $1 \leq b<a \leq n$. If $k=1$, then $S=X_{1}$ is a divisor chain. By [16], we have $\left(S^{e}\right) \mid\left[S^{e}\right]$. In what follows we let $k \geq 2$. Define $T_{i}:=\left\{\left(X_{i}, X_{j}\right): 1 \leq j<i\right\}$ for $2 \leq i \leq k$. For any $y_{s} \in S(1 \leq$ $s \leq n)$, define $n_{s}:=\left|\left\{2 \leq i \leq k: \max \left(T_{i}\right)=y_{s}\right\}\right|$. Clearly $n_{s}=0$ if $y_{s} \neq \max \left(T_{i}\right)$ for all $2 \leq i \leq k$. In particular, $n_{s}=0$ if $s \in A$ and thus $\sum_{s=1}^{n} n_{s}=\sum_{s \notin A} n_{s}=k-1$. We have the following results.

LEMMA 2.2 .

(i) For any integer $2 \leq i \leq k, T_{i}$ is a divisor chain.

(ii) For $1<a \leq k$, if $\max \left(T_{a}\right) \in X_{b}$, then $\left(X_{a}, X_{b}\right)=\max \left(T_{a}\right)$.

Proof. Since $\left(X_{i}, X_{j}\right) \mid \max \left(X_{i}\right)$ for all integers $j \geq 1$, by Lemma 2.1 we can easily see that $T_{i}$ is a divisor chain, proving (i). Clearly $\max \left(T_{a}\right) \mid\left(X_{a}, X_{b}\right)$ since $\max \left(T_{a}\right) \in X_{b}$. But $T_{a}$ is a divisor chain. So $\left(X_{a}, X_{b}\right) \mid \max \left(T_{a}\right)$ and hence $\left(X_{a}, X_{b}\right)=\max \left(T_{a}\right)$. This proves (ii).

Lemma 2.3. Let $1 \leq l \neq m \leq k, y_{t} \in X_{m}, y_{\alpha} \in X_{l}$ and $y_{\beta} \in S$.

(i) If $y_{\alpha} \nmid y_{t}$ and $y_{\alpha} \mid y_{\beta}$, then $\left(y_{\alpha}, y_{t}\right)=\left(y_{\beta}, y_{t}\right)$.

(ii) If $\left(X_{l}, y_{t}\right) \notin X_{l}$, then $\left(X_{l}, y_{t}\right)=\left(T_{l}, y_{t}\right)$.

(iii) If $y_{t} \nmid y_{\alpha}, y_{\alpha} \nmid y_{t}$ and $\left(y_{\alpha}, y_{t}\right)=y_{\omega}$, then $n_{\omega} \neq 0$.

Proof. (i) Let $\left(y_{\alpha}, y_{t}\right)=a \in S$ and $\left(y_{\beta}, y_{t}\right)=b \in S$. Clearly $y_{\alpha} \mid y_{\beta}$ and $a|b| y_{\beta}$. Since $\max _{x \in S}\left\{\left|G_{S}(x)\right|\right\}=1$, Lemma 2.1 applied to $\left\{b, y_{\alpha}, y_{\beta}\right\}$ tells us that either $b\left|y_{\alpha}\right| y_{\beta}$ or $y_{\alpha}|b| y_{\beta}$. If $y_{\alpha} \mid b=\left(y_{\beta}, y_{t}\right)$, then $y_{\alpha} \mid y_{t}$, contrary to assumption. So we must have $b \mid y_{\alpha}$ and $b \mid a$. Hence $\left(y_{\alpha}, y_{t}\right)=\left(y_{\beta}, y_{t}\right)$ as required.

(ii) Let $\left(X_{l}, y_{t}\right)=y_{t^{\prime}} \notin X_{l}$ and $\max \left(T_{l}\right) \in X_{l^{\prime}}$ for some positive integers $t^{\prime} \leq n$ and $l^{\prime}<l$. Then $y_{t^{\prime}} \in X_{l^{\prime \prime}}$ for some positive integer $l^{\prime \prime}<l$. We then derive that $y_{t^{\prime}}\left|\left(X_{l^{\prime \prime}}, X_{l}\right)\right| \max \left(T_{l}\right) \mid \max \left(X_{l^{\prime}}\right)$. By Lemma 2.2 we have

$$
\left(T_{l}, y_{t}\right)=\left(\left(X_{l}, X_{l^{\prime}}\right), y_{t}\right)=\left(\left(X_{l}, y_{t}\right), X_{l^{\prime}}\right)=\left(y_{t^{\prime}}, X_{l^{\prime}}\right)=y_{t^{\prime}}=\left(X_{l}, y_{t}\right) .
$$

(iii) Without loss of generality, we may let $l<m$. It suffices to show that $y_{\omega}=\max \left(T_{i}\right)$ for some $2 \leq i \leq k$. Let $y_{\omega} \in X_{r}$. Then $r \leq l<m$. Obviously $y_{\omega} \nmid \max \left(X_{i}\right)$ for $1 \leq i \leq r-1$ and $y_{\omega} \mid \max \left(X_{r}\right)$ as well as $y_{\omega} \mid \max \left(X_{m}\right)$. Thus we can define a nonempty index set $\left\{q_{1}, \ldots, q_{h}\right\}:=\{r+1 \leq q \leq k$ : $\left.y_{\omega} \mid \max \left(X_{q}\right)\right\}$. Clearly $m \in\left\{q_{1}, \ldots, q_{h}\right\}$.

We claim that there exists some $1 \leq j \leq h$ such that $\left(X_{q_{j}}, X_{r}\right)=y_{\omega}$. Since $y_{\alpha} \nmid y_{t}$ and $y_{t} \nmid \max \left(X_{l}\right)$, we have $y_{\omega}=\left(y_{\alpha}, y_{t}\right)=\left(X_{l}, y_{t}\right)=\left(X_{l}, X_{m}\right)$ by (i). So if $r=l$, the claim is true. If $r<l$, then $l \in\left\{q_{1}, \ldots, q_{h}\right\}$. Evidently $y_{\omega}\left|\left(X_{q_{j}}, X_{r}\right)\right| \max \left(X_{r}\right)$ for all $1 \leq j \leq h$. By Lemma 2.1 we 
know that $\left\{\left(X_{q_{1}}, X_{r}\right), \ldots,\left(X_{q_{h}}, X_{r}\right)\right\}$ is a divisor chain. Assume that the claim is not true. Then $\left(X_{q_{j}}, X_{r}\right)>y_{\omega}$ for all $1 \leq j \leq h$. So $\left(X_{l}, X_{m}\right) \geq$ $\left(\left(X_{l}, X_{r}\right),\left(X_{m}, X_{r}\right)\right)=\min \left(\left(X_{l}, X_{r}\right),\left(X_{m}, X_{r}\right)\right)>y_{\omega}$. This is absurd. The claim is proved.

Now let $i$ be the smallest $r+1 \leq q_{j} \leq k$ such that $\left(X_{q_{j}}, X_{r}\right)=y_{\omega}$. It remains to show that $y_{\omega}=\max \left(T_{i}\right)$. Since $y_{\omega}=\left(X_{i}, X_{r}\right)$, we have $y_{\omega} \mid \max \left(T_{i}\right)$. Let $\max \left(T_{i}\right) \in X_{v}$ for some $1 \leq v \leq i-1$. Then $y_{\omega} \mid \max \left(X_{v}\right)$ and so $v \in\left\{r, q_{1}, \ldots, q_{h}\right\}$. By Lemma 2.2 we have $\left(X_{i}, X_{v}\right)=\max \left(T_{i}\right)$. Let $\left(X_{v}, X_{r}\right)=y_{\omega^{\prime}}$. Suppose that $\max \left(T_{i}\right)>y_{\omega}$. Then $X_{v} \neq X_{r}$ and so $y_{\omega^{\prime}}>y_{\omega}$ by the minimality of $i$. Since $y_{\omega^{\prime}} \mid \max \left(X_{v}\right)$ and $\max \left(T_{i}\right) \mid \max \left(X_{v}\right)$, by Lemma 2.1 we have either $y_{\omega^{\prime}}\left|\max \left(T_{i}\right)\right| \max \left(X_{v}\right)$ or $\max \left(T_{i}\right)\left|y_{\omega^{\prime}}\right| \max \left(X_{v}\right)$. From this we deduce that $y_{\omega}=\left(X_{i}, X_{r}\right) \geq\left(\left(X_{i}, X_{v}\right),\left(X_{r}, X_{v}\right)\right)=\left(T_{i}, y_{\omega^{\prime}}\right)=$ $\min \left(\max \left(T_{i}\right), y_{\omega^{\prime}}\right)>y_{\omega}$, which is impossible. Thus $\max \left(T_{i}\right)=y_{\omega}$ as desired.

For any $s \in A$, we can define a unique integer $1 \leq l(s) \leq k$ such that $y_{s}=y_{a_{1}+\cdots+a_{l(s)}}=\max \left(X_{l(s)}\right)$. In the rest of this section, for any given $1 \leq t \leq n$, let $y_{t} \in X_{l\left(t^{\prime}\right)}$ and $y_{t^{\prime}}=\max \left(X_{l\left(t^{\prime}\right)}\right)$ for $1 \leq l\left(t^{\prime}\right) \leq k$. Then $t^{\prime} \in A$.

Lemma 2.4. Let $A_{1}:=\left\{s:\left(y_{s}, y_{t}\right) \notin X_{l(s)}, s \in A \backslash\left\{t^{\prime}\right\}\right\}$ and $A_{2}:=\{s:$ $\left.\left(y_{s}, y_{t}\right) \in X_{l(s)}, s \in A \backslash\left\{t^{\prime}\right\}\right\}$. Then

(i) $f_{1}(t):=\sum_{s \in A_{1}}\left(\left(y_{s}, y_{t}\right)^{e}-\left(T_{l(s)}, y_{t}\right)^{e}\right)=0$.

(ii) $f_{2}(t):=\sum_{s \in A_{2}}\left(y_{s}, y_{t}\right)^{e}-\sum_{s \in A_{2} \cup\left\{t^{\prime}\right\} \backslash\left\{a_{1}\right\}}\left(T_{l(s)}, y_{t}\right)^{e}=0$.

Proof. (i) If $s \in A_{1}$, then $\left(y_{s}, y_{t}\right) \notin X_{l(s)}$. By Lemma 2.3(ii) we have $\left(y_{s}, y_{t}\right)=\left(T_{l(s)}, y_{t}\right)$, and so $f_{1}=0$.

(ii) If $t^{\prime}=a_{1}$, then clearly $A_{2}=\emptyset$. Hence $f_{2}=0$ as required. Let now $t^{\prime} \neq a_{1}$. Consider the following two cases:

CASE 1: $t \in A$. Then $t=t^{\prime}$. Since $\left(X_{1}, y_{t}\right) \in X_{1}$, we can define a nonempty index set $\left\{t_{1}, \ldots, t_{r}\right\}:=\left\{1 \leq i \leq k:\left(X_{i}, y_{t}\right) \in X_{i}\right\}$, where $1=t_{1}<\cdots<t_{r}=l(t)$.

We assert that $\left(y_{t}, X_{t_{j-1}}\right)=\max \left(T_{t_{j}}\right) \in X_{t_{j-1}}$ for all $1<j \leq r$. Let $\left(y_{t}, X_{t_{j}}\right)=y_{t_{j}^{\prime}} \in X_{t_{j}}$ for $1<j \leq r$. Clearly there exists a unique $1 \leq$ $b(j)<t_{j}$ such that $\max \left(T_{t_{j}}\right) \in X_{b(j)}$. By Lemmas 2.2 and 2.3(i), we have $\left(y_{t_{j}^{\prime}}, X_{b(j)}\right)=\left(X_{t_{j}}, X_{b(j)}\right)=\max \left(T_{t_{j}}\right) \in X_{b(j)}$. Then $\left(y_{t_{j}^{\prime}}, X_{b(j)}\right)\left|\left(y_{t}, X_{b(j)}\right)\right|$ $\max \left(X_{b(j)}\right)$ and so $\left(y_{t}, X_{b(j)}\right) \in X_{b(j)}$. Therefore $X_{b(j)} \in\left\{X_{t_{1}}, \ldots, X_{t_{j-1}}\right\}$. Since $y_{t_{j}^{\prime}} \mid y_{t}$ and $\left(y_{t}, X_{t_{j-1}}\right)=y_{t_{j-1}^{\prime}} \mid y_{t}$, by Lemma 2.1 we have $y_{t_{j-1}^{\prime}}\left|y_{t_{j}^{\prime}}\right| y_{t}$. Then $y_{t_{j-1}^{\prime}}=\left(y_{t_{j}^{\prime}}, y_{t_{j-1}^{\prime}}\right)\left|\left(X_{t_{j}}, X_{t_{j-1}}\right)\right| \max \left(T_{t_{j}}\right) \mid \max \left(X_{t_{j}}\right)$. Since $y_{t_{j-1}^{\prime}} \in$ $X_{t_{j-1}}$, we have $\max \left(T_{t_{j}}\right) \in X_{t_{j-1}}$. Clearly $y_{t_{j}^{\prime}} \nmid X_{t_{j-1}}$ for all $1<j \leq r$. Then 
$\left(y_{t}, X_{t_{j-1}}\right)=\left(y_{t_{j}^{\prime}}, X_{t_{j-1}}\right)=\left(X_{t_{j}}, X_{t_{j-1}}\right)=\max \left(T_{t_{j}}\right)$ by Lemmas $2.3(\mathrm{i})$ and 2.2. The assertion is proved.

For each $s \in A_{2}$, we can find a unique $1 \leq j<r$ such that $y_{s} \in X_{t_{j}}$. Note that $\max \left(T_{t_{j}}\right) \mid y_{t}$ for all $1 \leq j \leq r$. Therefore

$$
\begin{aligned}
f_{2} & =\sum_{1 \leq j<r}\left(X_{t_{j}}, y_{t}\right)^{e}-\sum_{1<j \leq r}\left(T_{t_{j}}, y_{t}\right)^{e} \\
& =\sum_{1 \leq j<r} \max \left(T_{t_{j+1}}\right)^{e}-\sum_{1<j \leq r} \max \left(T_{t_{j}}\right)^{e}=0 .
\end{aligned}
$$

CASe 2: $t \notin A$. If $l(s) \in A_{2}$, then $l(s)<l\left(t^{\prime}\right)$ and $y_{t} \nmid y_{s}$. Then $\left(y_{s}, y_{t}\right)=$ $\left(y_{s}, y_{t^{\prime}}\right)$ by Lemma 2.3(i). Since $y_{t} \nmid y_{s}$ and $y_{t} \nmid \max \left(T_{l(s)}\right)$, by Lemma 2.3(i) we have $\left(T_{l(s)}, y_{t}\right)=\left(T_{l(s)}, y_{t^{\prime}}\right)$ for all $l(s)<l\left(t^{\prime}\right)$. Then

$$
f_{2}=\sum_{s \in A_{2}}\left(y_{s}, y_{t^{\prime}}\right)^{e}-\sum_{s \in A_{2} \cup\left\{t^{\prime}\right\} \backslash\left\{a_{1}\right\}}\left(T_{l(s)}, y_{t^{\prime}}\right)^{e}=0 .
$$

Let $A_{1}^{\prime}=\left\{s:\left(y_{s}, y_{t^{\prime}}\right) \notin X_{l(s)}, s \in A \backslash\left\{t^{\prime}\right\}\right\}$ and $A_{2}^{\prime}=\left\{s:\left(y_{s}, y_{t^{\prime}}\right) \in\right.$ $\left.X_{l(s)}, s \in A \backslash\left\{t^{\prime}\right\}\right\}$. It is easy to see that $A_{1}^{\prime}=A_{1}$ and $A_{2}^{\prime}=A_{2}$. If we replace $A_{2}$ by $A_{2}^{\prime}$ and $t$ by $t^{\prime}$, Case 1 gives $f_{2}=0$.

Definition. Define a matrix $C:=\left(c_{s t}\right) \in M_{n}(\mathbb{Z})$, where

$$
c_{s t}=\frac{y_{t}^{e}}{\left(y_{s}, y_{t}\right)^{e}} \delta_{s t}
$$

and $\delta_{s t}$ is defined by: $\delta_{s 1}=1$ if $s \in A ;-n_{s}$ if $s \notin A, \delta_{1 t}=1-n_{1}$ if $t>1$, and for $s, t>1$,

$$
\underbrace{\delta_{s t}}_{\text {if } t \in A}=\{\begin{array}{ll}
1, & s \in A \backslash\{t\}, \\
-n_{s}, & \text { otherwise, }
\end{array} \quad \underbrace{\delta_{s t}}_{\text {if } t \notin A}= \begin{cases}-1-n_{s}, & s=t, \\
1, & s \in A, \\
-n_{s}, & \text { otherwise. }\end{cases}
$$

Now we state the first main result of this paper as follows.

THEOREM 2.5 .

(i) Let $S$ be a gcd-closed set such that $\max _{x \in S}\left\{\left|G_{S}(x)\right|\right\}=1$. Then $\left(S^{e}\right)^{-1}\left[S^{e}\right]=C$, where $C \in M_{n}(\mathbb{Z})$ is defined as above. In particular, Conjecture 1.1 holds.

(ii) For each integer $r \geq 2$, there exists a gcd-closed set $S$ such that $\max _{x \in S}\left\{\left|G_{S}(x)\right|\right\}=r$ and the power GCD matrix $\left(S^{e}\right)$ on $S$ does not divide the power $L C M$ matrix $\left[S^{e}\right]$ on $S$ in $M_{n}(\mathbb{Z})$.

Proof. (i) First note that $S$ is as in (1). Then $S=\left\{y_{1}, \ldots, y_{n}\right\}$ with $y_{1}=1$. In what follows we show $\left[S^{e}\right]=\left(S^{e}\right) C$, i.e. $\left[y_{m}, y_{t}\right]^{e}=\sum_{s=1}^{n}\left(y_{m}, y_{s}\right)^{e} c_{s t}$ for all $1 \leq m, t \leq n$. Let $y_{m} \in X_{l\left(m^{\prime}\right)}$ and $y_{m^{\prime}}=\max \left(X_{l\left(m^{\prime}\right)}\right)$ for $1 \leq l\left(m^{\prime}\right) \leq k$ and $m^{\prime} \in A$. Let $\left(y_{m}, y_{t}\right)=y_{u} \in X_{l\left(u^{\prime}\right)}$ and $y_{u^{\prime}}=\max \left(X_{l\left(u^{\prime}\right)}\right)$. Consider the following three cases: 
Case 1: $t=1$. We have

where

$$
\sum_{s=1}^{n}\left(y_{m}, y_{s}\right)^{e} c_{s 1}=\sum_{s \in A}\left(y_{m}, y_{s}\right)^{e}-\sum_{s \notin A} n_{s}\left(y_{m}, y_{s}\right)^{e}=y_{m}^{e}+\Delta,
$$

$$
\Delta=\sum_{s \in A \backslash\left\{m^{\prime}\right\}}\left(y_{m}, y_{s}\right)^{e}-\sum_{s \in A \backslash\left\{a_{1}\right\}}\left(y_{m}, T_{l(s)}\right)^{e} .
$$

Clearly $\Delta=f_{1}(m)+f_{2}(m)=0$ by Lemma 2.4. Thus $\sum_{s=1}^{n}\left(y_{m}, y_{s}\right)^{e} c_{s 1}=$ $y_{m}^{e}=\left[y_{m}, y_{1}\right]^{e}$.

Case 2: $t \in A$. We have

$$
\begin{aligned}
\sum_{s=1}^{n}\left(y_{m}, y_{s}\right)^{e} c_{s t}= & \left(y_{m}, y_{1}\right)^{e} y_{t}^{e}\left(1-n_{1}\right)+\left(y_{m}, y_{t}\right)^{e}\left(-n_{t}\right) \\
& +\sum_{s \in A \backslash\{t\}} \frac{\left(y_{m}, y_{s}\right)^{e} y_{t}^{e}}{\left(y_{s}, y_{t}\right)^{e}}-\sum_{s \notin A, s \neq 1} \frac{n_{s}\left(y_{m}, y_{s}\right)^{e} y_{t}^{e}}{\left(y_{s}, y_{t}\right)^{e}} \\
= & y_{t}^{e}+\sum_{s \in A \backslash\{t\}} \frac{\left(y_{m}, y_{s}\right)^{e} y_{t}^{e}}{\left(y_{s}, y_{t}\right)^{e}}-\sum_{s \notin A} \frac{n_{s}\left(y_{m}, y_{s}\right)^{e} y_{t}^{e}}{\left(y_{s}, y_{t}\right)^{e}}=y_{t}^{e}+y_{t}^{e} g_{1},
\end{aligned}
$$

since $n_{t}=0$ for $t \in A$, where

$$
g_{1}=\sum_{s \in A \backslash\{t\}} \frac{\left(y_{m}, y_{s}\right)^{e}}{\left(y_{s}, y_{t}\right)^{e}}-\sum_{s \in A \backslash\left\{a_{1}\right\}} \frac{\left(T_{l(s)}, y_{m}\right)^{e}}{\left(T_{l(s)}, y_{t}\right)^{e}} .
$$

Let $A_{1}$ and $A_{2}$ be as in Lemma 2.4 , and let $\left\{t_{1}, \ldots, t_{r}\right\}$ be as in the proof of Lemma 2.4. Consider the following two subcases.

SubCASE 2-1: $y_{m} \mid y_{t}$. Since $\left[y_{m}, y_{t}\right]=y_{t}$, it suffices to show $g_{1}=0$. We have

$$
\begin{aligned}
g_{1}= & \left(\sum_{s \in A_{1}} \frac{\left(y_{s}, y_{m}\right)^{e}}{\left(y_{s}, y_{t}\right)^{e}}-\sum_{s \in A_{1}} \frac{\left(T_{l(s)}, y_{m}\right)^{e}}{\left(T_{l(s)}, y_{t}\right)^{e}}\right) \\
& +\left(\sum_{s \in A_{2}} \frac{\left(y_{s}, y_{m}\right)^{e}}{\left(y_{s}, y_{t}\right)^{e}}-\sum_{s \in A_{2} \cup\{t\} \backslash\left\{a_{1}\right\}} \frac{\left(T_{l(s)}, y_{m}\right)^{e}}{\left(T_{l(s)}, y_{t}\right)^{e}}\right) \\
= & \sum_{s \in A_{1}}\left(\frac{\left(y_{s}, y_{m}\right)^{e}}{\left(y_{s}, y_{t}\right)^{e}}-\frac{\left(T_{l(s)}, y_{m}\right)^{e}}{\left(T_{l(s)}, y_{t}\right)^{e}}\right)+\left(\sum_{1 \leq j<r} \frac{\left(X_{t_{j}}, y_{m}\right)^{e}}{\left(X_{t_{j}}, y_{t}\right)^{e}}-\sum_{1<j \leq r} \frac{\left(T_{t_{j}}, y_{m}\right)^{e}}{\left(T_{t_{j}}, y_{t}\right)^{e}}\right) \\
= & \sum_{s \in A_{1}}\left(\frac{\left(y_{s}, y_{m}\right)^{e}}{\left(y_{s}, y_{t}\right)^{e}}-\frac{\left(T_{l(s)}, y_{m}\right)^{e}}{\left(T_{l(s)}, y_{t}\right)^{e}}\right)+\sum_{1<j \leq r}\left(\frac{\left(X_{t_{j-1}}, y_{m}\right)^{e}}{\left(X_{t_{j-1}}, y_{t}\right)^{e}}-\frac{\left(T_{t_{j}}, y_{m}\right)^{e}}{\left(T_{t_{j}}, y_{t}\right)^{e}}\right) \\
= & : h_{1}+h_{2} .
\end{aligned}
$$

If $s \in A_{1}$, then $\left(y_{t}, y_{s}\right) \notin X_{l(s)}$. Since $\left(y_{m}, y_{s}\right) \mid\left(y_{t}, y_{s}\right)$ we have $\left(y_{m}, y_{s}\right) \notin$ $X_{l(s)}$. From Lemma 2.3(ii), we deduce $\left(y_{s}, y_{m}\right)=\left(T_{l(s)}, y_{m}\right)$ and $\left(y_{s}, y_{t}\right)=$ $\left(T_{l(s)}, y_{t}\right)$. So $h_{1}=0$. 
We now show $h_{2}=0$. We have proved in Lemma 2.4 that $\left(y_{t}, X_{t_{j-1}}\right)=$ $\max \left(T_{t_{j}}\right) \in X_{t_{j-1}}$ for all $1<j \leq r$. Let first $m^{\prime}=a_{1}$, i.e. $y_{m} \in X_{1}$. For $2<j \leq r$, we have $\max \left(T_{t_{j}}\right) \nmid y_{m}$. Then by Lemma 2.3(i), $\left(T_{t_{j}}, y_{m}\right)=$ $\left(X_{t_{j-1}}, y_{m}\right)$. Let $j=2$. Then $y_{m} \mid\left(y_{t}, y_{m^{\prime}}\right)=\max \left(T_{t_{2}}\right) \in X_{1}$ and so $\left(T_{t_{2}}, y_{m}\right)$ $=y_{m}=\left(X_{1}, y_{m}\right)$. Thus $h_{2}=0$. Let now $m^{\prime} \neq a_{1}$. Since $y_{m}\left|\left(y_{t}, y_{m^{\prime}}\right)\right| y_{m^{\prime}}$ and $y_{m}, y_{m^{\prime}} \in X_{l\left(m^{\prime}\right)}$, we have $\left(y_{m^{\prime}}, y_{t}\right) \in X_{l\left(m^{\prime}\right)}$, which implies that $l\left(m^{\prime}\right) \in$ $\left\{t_{1}, \ldots, t_{r}\right\}$. Write $l\left(m^{\prime}\right)=t_{j_{0}}$ for some $1 \leq j_{0} \leq r$. Since $\left(y_{t}, X_{t_{j-1}}\right)=$ $\max \left(T_{t_{j}}\right) \in X_{t_{j-1}}$ for all $1<j \leq r$, we have $\max \left(T_{t_{j}}\right) \nmid y_{m}$ and $\left(X_{t_{j-1}}, y_{m}\right)=$ $\left(T_{t_{j}}, y_{m}\right)$ for all $j_{0}+2 \leq j \leq r$ by Lemma $2.3(\mathrm{i})$. For all $1<j \leq j_{0}$ we have $y_{m} \nmid \max \left(T_{t_{j}}\right)$ and so $\left(X_{t_{j-1}}, y_{m}\right)=\left(X_{t_{j-1}}, y_{t}\right)=\max \left(T_{t_{j}}\right)$ by Lemma 2.3(i). Hence $\left(X_{t_{j-1}}, y_{m}\right)=\left(T_{t_{j}}, y_{m}\right)$ for all $1<j \leq j_{0}$. For $j=j_{0}+1$, we have $\left(T_{t_{j_{0}+1}}, y_{m}\right)=\left(\left(y_{t}, X_{t_{j_{0}}}\right), y_{m}\right)=\left(X_{t_{j_{0}}}, y_{m}\right)$. This implies that $h_{2}=0$, which means $g_{1}=0$. (Note that

$$
g_{1}^{\prime}:=\sum_{s \in A \backslash\{t\}} \frac{\left(y_{t}, y_{s}\right)^{e}}{\left(y_{s}, y_{m}\right)^{e}}-\sum_{s \in A \backslash\left\{a_{1}\right\}} \frac{\left(T_{l(s)}, y_{t}\right)^{e}}{\left(T_{l(s)}, y_{m}\right)^{e}}=0,
$$

which will be used in Subcase 3-2).

Subcase 2-2: $y_{m} \nmid y_{t}$. Clearly $m^{\prime} \neq t$. Since $\left(y_{m}, y_{t}\right)=y_{u}$ and $y_{t} \nmid y_{m}$ for all $y_{m} \in S$, we have $n_{u} \neq 0$ by Lemma 2.3(iii). So we can find a $v \in A$ such that $y_{u}=\max \left(T_{l(v)}\right)$. Then $\left(T_{l(v)}, y_{m}\right) /\left(T_{l(v)}, y_{t}\right)=y_{u} / y_{u}=1$. Since $y_{u}\left|\left(y_{t}, y_{u^{\prime}}\right)\right| y_{u^{\prime}}$, we have $\left(y_{t}, y_{u^{\prime}}\right) \in X_{l\left(u^{\prime}\right)}$ and so $l\left(u^{\prime}\right) \in\left\{t_{1}, \ldots, t_{r}\right\}$. Let $t_{j_{1}}=l\left(u^{\prime}\right)$ for some $1 \leq j_{1} \leq r$. By Lemma 2.3(i) and $y_{m} \nmid y_{t}$, we have $\left(y_{m}, y_{m^{\prime}}\right) /\left(y_{m^{\prime}}, y_{t}\right)=y_{m} /\left(y_{m}, y_{t}\right)$. Then

$$
\sum_{s=1}^{n}\left(y_{m}, y_{s}\right)^{e} c_{s t}=\left[y_{m}, y_{t}\right]^{e}+y_{t}^{e} g_{2},
$$

where

$$
g_{2}=\sum_{s \in A \backslash\left\{t, m^{\prime}\right\}} \frac{\left(y_{s}, y_{m}\right)^{e}}{\left(y_{s}, y_{t}\right)^{e}}-\sum_{s \in A \backslash\left\{a_{1}, v\right\}} \frac{\left(T_{l(s)}, y_{m}\right)^{e}}{\left(T_{l(s)}, y_{t}\right)^{e}} .
$$

In what follows we show that $g_{2}=0$.

Subcase 2-2-1: $m^{\prime} \in A_{2}$, i.e. $\left(y_{m^{\prime}}, y_{t}\right) \in X_{l\left(m^{\prime}\right)}$. Then $l\left(u^{\prime}\right) \neq l(t)=t_{r}$. Since $y_{m} \nmid y_{t}$, we have $y_{u}=\left(y_{m}, y_{t}\right)=\left(y_{m^{\prime}}, y_{t}\right) \in X_{l\left(m^{\prime}\right)}$ by Lemma 2.3(i). Then $l\left(m^{\prime}\right)=l\left(u^{\prime}\right)=t_{j_{1}}$. Since $\left(y_{m^{\prime}}, y_{t}\right)=\max \left(T_{t_{j_{1}+1}}\right)$, we may let $l(v)=$ $t_{j_{1}+1}$. Note that $\left\{l(s): s \in A_{2}\right\}=\left\{t_{1}, \ldots, t_{r-1}\right\}$. Then

$$
\begin{aligned}
g_{2}= & \sum_{s \in A_{1}}\left(\frac{\left(y_{s}, y_{m}\right)^{e}}{\left(y_{s}, y_{t}\right)^{e}}-\frac{\left(T_{l(s)}, y_{m}\right)^{e}}{\left(T_{l(s)}, y_{t}\right)^{e}}\right)+\sum_{s \in A_{2} \backslash\left\{m^{\prime}\right\}} \frac{\left(y_{s}, y_{m}\right)^{e}}{\left(y_{s}, y_{t}\right)^{e}} \\
& -\sum_{s \in A_{2} \cup\{t\} \backslash\left\{a_{1}, v\right\}} \frac{\left(T_{l(s)}, y_{m}\right)^{e}}{\left(T_{l(s)}, y_{t}\right)^{e}}
\end{aligned}
$$




$$
\begin{aligned}
= & \sum_{s \in A_{1}}\left(\frac{\left(y_{s}, y_{m}\right)^{e}}{\left(y_{s}, y_{t}\right)^{e}}-\frac{\left(T_{l(s)}, y_{m}\right)^{e}}{\left(T_{l(s)}, y_{t}\right)^{e}}\right)+\sum_{1<j \leq j_{1}}\left(\frac{\left(X_{t_{j-1}}, y_{m}\right)^{e}}{\left(X_{t_{j-1}}, y_{t}\right)^{e}}-\frac{\left(T_{t_{j}}, y_{m}\right)^{e}}{\left(T_{t_{j}}, y_{t}\right)^{e}}\right) \\
& +\sum_{j_{1}+2 \leq j \leq r}\left(\frac{\left(X_{t_{j-1}}, y_{m}\right)^{e}}{\left(X_{t_{j-1}}, y_{t}\right)^{e}}-\frac{\left(T_{t_{j}}, y_{m}\right)^{e}}{\left(T_{t_{j}}, y_{t}\right)^{e}}\right)=: h_{1}+h_{3}+h_{4} .
\end{aligned}
$$

For $1<j \leq j_{1}-1$, since $\max \left(T_{t_{j}}\right) \in X_{t_{j-1}}, \max \left(T_{t_{j_{1}+1}}\right) \nmid \max \left(X_{t_{j-1}}\right)$ and $\left(y_{m}, y_{t}\right)=\max \left(T_{t_{j_{1}+1}}\right)$, we have

$$
\left(X_{t_{j-1}}, y_{m}\right)=\left(X_{t_{j-1}}, T_{t_{j_{1}+1}}\right)=\left(X_{t_{j-1}}, y_{t}\right)=\max \left(T_{t_{j}}\right)=\left(T_{t_{j}}, y_{m}\right)
$$

by Lemma $2.3(\mathrm{i})$. But by Lemmas 2.2 and $2.3(\mathrm{i}),\left(X_{t_{j_{1}-1}}, y_{m}\right)=\left(X_{t_{j_{1}-1}}, y_{m^{\prime}}\right)$ $=\max \left(T_{t_{j_{1}}}\right)=\left(T_{t_{j_{1}}}, y_{m}\right)$. Since $\max \left(T_{t_{j}}\right) \in X_{t_{j-1}}$ and $\max \left(T_{t_{j}}\right) \nmid y_{m}$ for $j_{1}+2 \leq j \leq r$, we have $\left(X_{t_{j-1}}, y_{m}\right)=\left(T_{t_{j}}, y_{m}\right)$ by Lemma 2.3(i). Since $\left(y_{t}, X_{t_{j-1}}\right)=\max \left(T_{t_{j}}\right)$ for all $1<j \leq r$, we have $h_{3}=h_{4}=0$.

Now we treat $h_{1}$. Let first $s>m^{\prime} \geq m$ and $s \in A_{1}$. Then $\left(y_{s}, y_{m}\right) \notin X_{l(s)}$ for all $s \in A_{1}$ and so $\left(y_{s}, y_{m}\right)=\left(T_{l(s)}, y_{m}\right)$ by Lemma 2.3(ii). Let $s<m \leq$ $m^{\prime}$ and $s \in A_{1}$. Clearly $\max \left(T_{t_{j_{1}+1}}\right) \nmid \max \left(X_{l(s)}\right)=y_{s}$. Then $\left(y_{s}, y_{m}\right)=$ $\left(y_{s}, T_{t_{j_{1}+1}}\right)=\left(y_{s}, y_{t}\right) \notin X_{l(s)}$ by Lemma $2.3(\mathrm{i})$. Hence $\left(y_{s}, y_{m}\right)=\left(T_{l(s)}, y_{m}\right)$ by Lemma $2.3\left(\right.$ ii). Since $\left(y_{s}, y_{t}\right)=\left(T_{l(s)}, y_{t}\right)$ for all $s \in A_{1}$, we have $h_{1}=0$. Thus $g_{2}=h_{1}+h_{3}+h_{4}=0$.

Subcase 2-2-2: $m^{\prime} \in A_{1}$, i.e. $y_{u}=\left(y_{m^{\prime}}, y_{t}\right) \notin X_{l\left(m^{\prime}\right)}$. Define an index set $B:=\left\{s \in A_{1}:\left(y_{m}, y_{s}\right) \notin X_{l(s)}\right\}$. If $\left(y_{m}, y_{t}\right) \in X_{l(t)}$, i.e. $l\left(u^{\prime}\right)=l(t)$, then let $\left\{m_{r}, \ldots, m_{w}\right\}:=\left\{i: l(t) \leq i \leq k,\left(X_{i}, X_{l\left(m^{\prime}\right)}\right) \in X_{i}\right\}$, where $l(t)=t_{r}=$ $m_{r}<\cdots<m_{w}=l\left(m^{\prime}\right)$. As in the proof of $\left(y_{t}, X_{t_{j-1}}\right)=\max \left(T_{t_{j}}\right) \in X_{t_{j-1}}$ for all $1<j \leq r$, we can show $\left(y_{m^{\prime}}, X_{m_{j-1}}\right)=\max \left(T_{m_{j}}\right) \in X_{m_{j-1}}$ for all $r<j \leq w$. Then $y_{u}=\left(y_{m^{\prime}}, y_{t}\right)=\max \left(T_{m_{r+1}}\right)$. Hence we may let $l(v)=m_{r+1}$. Since $\left\{l(s): s \in A_{1} \backslash B\right\}=\left\{m_{r+1}, \ldots, m_{w}\right\}$ and $\{l(s): s \in$ $\left.A_{2}\right\}=\left\{t_{1}, \ldots, t_{r-1}\right\}$, we have

$$
\begin{aligned}
g_{2}= & \sum_{s \in B}\left(\frac{\left(y_{s}, y_{m}\right)^{e}}{\left(y_{s}, y_{t}\right)^{e}}-\frac{\left(T_{l(s)}, y_{m}\right)^{e}}{\left(T_{l(s)}, y_{t}\right)^{e}}\right)+\sum_{1<j \leq r}\left(\frac{\left(X_{t_{j-1}}, y_{m}\right)^{e}}{\left(X_{t_{j-1}}, y_{t}\right)^{e}}-\frac{\left(T_{t_{j}}, y_{m}\right)^{e}}{\left(T_{t_{j}}, y_{t}\right)^{e}}\right) \\
& +\sum_{r+2 \leq j \leq w}\left(\frac{\left(X_{m_{j-1}}, y_{m}\right)^{e}}{\left(X_{m_{j-1}}, y_{t}\right)^{e}}-\frac{\left(T_{m_{j}}, y_{m}\right)^{e}}{\left(T_{m_{j}}, y_{t}\right)^{e}}\right)=: h_{5}+h_{6}+h_{7} .
\end{aligned}
$$

If $s \in B$, we have $\left(y_{s}, y_{t}\right)=\left(T_{l(s)}, y_{t}\right)$ and $\left(y_{s}, y_{m}\right)=\left(T_{l(s)}, y_{m}\right)$ by Lemma 2.3(ii). Then $h_{5}=0$. Since for all $1<j \leq r, y_{u} \nmid \max \left(T_{t_{j}}\right)$ and $y_{u} \nmid$ $\max \left(X_{t_{j-1}}\right)$, by Lemma $2.3(\mathrm{i})$ we have $\left(X_{t_{j-1}}, y_{t}\right)=\left(X_{t_{j-1}}, y_{u}\right)=\left(X_{t_{j-1}}, y_{m}\right)$ and $\left(T_{t_{j}}, y_{t}\right)=\left(T_{t_{j}}, y_{u}\right)=\left(T_{t_{j}}, y_{m}\right)$. Hence $h_{6}=0$. As in the proof of $h_{4}=0$, we can show $h_{7}=0$. Thus $g_{2}=0$.

If $\left(y_{m}, y_{t}\right) \notin X_{l(t)}$, then let $\left\{m_{j_{1}}, m_{j_{1}+1}, \ldots, m_{w^{\prime}}\right\}:=\left\{i: l\left(u^{\prime}\right)=m_{j_{1}} \leq\right.$ $\left.i \leq k,\left(X_{i}, X_{l\left(m^{\prime}\right)}\right) \in X_{i}\right\}$, where $l\left(u^{\prime}\right)=m_{j_{1}}<m_{j_{1}+1}<\cdots<m_{w^{\prime}}=l\left(m^{\prime}\right)$. Clearly $l\left(u^{\prime}\right) \neq t_{r}$. Since $m^{\prime}, t \in A$, we have $y_{u}\left|\left(y_{m^{\prime}}, X_{m_{j_{1}}}\right)=\max \left(T_{m_{j_{1}+1}}\right)\right| y_{u^{\prime}}$ 
and $y_{u}\left|\left(y_{m^{\prime}}, X_{t_{j_{1}}}\right)=\max \left(T_{t_{j_{1}+1}}\right)\right| y_{u^{\prime}}$. Then either $y_{u}\left|\max \left(T_{m_{j_{1}+1}}\right)\right|$ $\max \left(T_{t_{j_{1}+1}}\right) \mid y_{u^{\prime}}$ or $y_{u}\left|\max \left(T_{t_{j_{1}+1}}\right)\right| \max \left(T_{m_{j_{1}+1}}\right) \mid y_{u^{\prime}}$ by Lemma 2.1. But

$$
y_{u}=\left(y_{m^{\prime}}, y_{t}\right) \geq\left(\left(y_{m^{\prime}}, y_{u}^{\prime}\right),\left(y_{t}, y_{u^{\prime}}\right)\right)=\left(\max \left(T_{m_{j_{1}+1}}\right), \max \left(T_{t_{j_{1}+1}}\right)\right) .
$$

Then $y_{u}=\min \left(\max \left(T_{m_{j_{1}+1}}\right), \max \left(T_{t_{j_{1}+1}}\right)\right)$. So either $y_{u}=\max \left(T_{m_{j_{1}+1}}\right)$ or $y_{u}=\max \left(T_{t_{j_{1}+1}}\right)$. Then we may let $l(v)=t_{j_{1}+1}$ or $l(v)=m_{j_{1}+1}$. Note that $\left\{l(s): s \in A_{1} \backslash B\right\}=\left\{m_{j_{1}+1}, \ldots, m_{w^{\prime}}\right\}$ and $\left\{l(s): s \in A_{2}\right\}=\left\{t_{1}, \ldots, t_{r-1}\right\}$. If $l(v)=t_{j_{1}+1}$, then

$$
\begin{aligned}
g_{2}= & \sum_{1<j \leq j_{1}}\left(\frac{\left(X_{t_{j-1}}, y_{m}\right)^{e}}{\left(X_{t_{j-1}}, y_{t}\right)^{e}}-\frac{\left(T_{t_{j}}, y_{m}\right)^{e}}{\left(T_{t_{j}}, y_{t}\right)^{e}}\right)+\sum_{j_{1}+2 \leq j \leq r}\left(\frac{\left(X_{t_{j-1}}, y_{m}\right)^{e}}{\left(X_{t_{j-1}}, y_{t}\right)^{e}}-\frac{\left(T_{t_{j}}, y_{m}\right)^{e}}{\left(T_{t_{j}}, y_{t}\right)^{e}}\right) \\
& +\sum_{j_{1}+1 \leq j \leq w^{\prime}}\left(\frac{\left(X_{m_{j-1}}, y_{m}\right)^{e}}{\left(X_{m_{j-1}}, y_{t}\right)^{e}}-\frac{\left(T_{m_{j}}, y_{m}\right)^{e}}{\left(T_{m_{j}}, y_{t}\right)^{e}}\right)+h_{5}:=h_{3}+h_{4}+h_{8}+h_{5} .
\end{aligned}
$$

As in Subcase 2-2-1, we can prove $h_{3}=0$. As in the proof of $h_{6}=0$, we can show $h_{4}=h_{8}=0$. Notice that $h_{5}=0$. Thus $g_{2}=0$. Similarly, we can show that if $l(v)=m_{j_{1}+1}$, then $g_{2}=0$. Therefore Case 2 is proved.

Case 3: $t \notin A$. We have

$$
\begin{aligned}
\sum_{s=1}^{n}\left(y_{m}, y_{s}\right)^{e} c_{s t}= & y_{t}^{e}\left(1-n_{1}\right)-\left(1+n_{t}\right)\left(y_{t}, y_{m}\right)^{e} \\
& +\sum_{s \in A} \frac{\left(y_{m}, y_{s}\right)^{e} y_{t}^{e}}{\left(y_{s}, y_{t}\right)^{e}}-\sum_{s \notin A, s \neq 1, t} \frac{n_{s}\left(y_{m}, y_{s}\right)^{e} y_{t}^{e}}{\left(y_{s}, y_{t}\right)^{e}} \\
= & \left(y_{t}^{e}-\left(y_{t}, y_{m}\right)^{e}\right)+\sum_{s \in A} \frac{\left(y_{m}, y_{s}\right)^{e} y_{t}^{e}}{\left(y_{s}, y_{t}\right)^{e}}-\sum_{s \notin A} \frac{n_{s}\left(y_{m}, y_{s}\right)^{e} y_{t}^{e}}{\left(y_{s}, y_{t}\right)^{e}} .
\end{aligned}
$$

Consider the following three subcases.

Subcase 3-1: $y_{m} \mid y_{t}$. We have

$$
\begin{aligned}
\sum_{s=1}^{n}\left(y_{m}, y_{s}\right)^{e} c_{s t} & =y_{t}^{e}+y_{t}^{e}\left(\sum_{s \in A \backslash\left\{t^{\prime}\right\}} \frac{\left(y_{s}, y_{m}\right)^{e}}{\left(y_{s}, y_{t}\right)^{e}}-\sum_{s \in A \backslash\left\{a_{1}\right\}} \frac{\left(T_{l(s)}, y_{m}\right)^{e}}{\left(T_{l(s)}, y_{t}\right)^{e}}\right) \\
& =y_{t}^{e}+y_{t}^{e}\left(h_{1}+h_{9}\right),
\end{aligned}
$$

where

$$
h_{9}=\sum_{s \in A_{2}} \frac{\left(y_{s}, y_{m}\right)^{e}}{\left(y_{s}, y_{t}\right)^{e}}-\sum_{s \in A_{2} \cup\left\{t^{\prime}\right\} \backslash\left\{a_{1}\right\}} \frac{\left(T_{l(s)}, y_{m}\right)^{e}}{\left(T_{l(s)}, y_{t}\right)^{e}} .
$$

As in Subcase 2-1, we have $h_{1}=0$ since $t$ is independent of $t \in A$. If $s \in A_{2}$, then $y_{t} \nmid y_{s}$ and $y_{t} \nmid \max \left(T_{l(s)}\right)$. By Lemma 2.3(i), $\left(y_{s}, y_{t}\right)=\left(y_{s}, y_{t^{\prime}}\right)$ and 
$\left(T_{l(s)}, y_{t}\right)=\left(T_{l(s)}, y_{t^{\prime}}\right)$. Thus

$$
h_{9}=\sum_{s \in A_{2}} \frac{\left(y_{s}, y_{m}\right)^{e}}{\left(y_{s}, y_{t^{\prime}}\right)^{e}}-\sum_{s \in A_{2} \cup\left\{t^{\prime}\right\} \backslash\left\{a_{1}\right\}} \frac{\left(T_{l(s)}, y_{m}\right)^{e}}{\left(T_{l(s)}, y_{t^{\prime}}\right)^{e}} .
$$

Since $t^{\prime} \in A, h_{2}=0$ gives $h_{9}=0$.

SubCASE 3-2: $y_{t} \mid y_{m}$. We have

$$
\begin{aligned}
\sum_{s=1}^{n}\left(y_{m}, y_{s}\right)^{e} c_{s t} & =y_{m}^{e}+y_{t}^{e}\left(\sum_{s \in A \backslash\left\{m^{\prime}\right\}} \frac{\left(y_{s}, y_{m}\right)^{e}}{\left(y_{s}, y_{t}\right)^{e}}-\sum_{s \in A \backslash\left\{a_{1}\right\}} \frac{\left(T_{l(s)}, y_{m}\right)^{e}}{\left(T_{l(s)}, y_{t}\right)^{e}}\right) \\
& =: y_{m}^{e}+y_{t}^{e} g_{3} .
\end{aligned}
$$

Let

$$
g_{3}^{\prime}=\sum_{s \in A \backslash\left\{m^{\prime}\right\}} \frac{\left(y_{s}, y_{t}\right)^{e}}{\left(y_{s}, y_{m}\right)^{e}}-\sum_{s \in A \backslash\left\{a_{1}\right\}} \frac{\left(T_{l(s)}, y_{t}\right)^{e}}{\left(T_{l(s)}, y_{m}\right)^{e}} .
$$

Since $y_{t} \mid y_{m}, h_{1}+h_{9}=0$ in Subcase 3-1 gives $g_{3}^{\prime}=0$. Then as in Subcase $2-1, g_{1}=0$ implying $g_{1}^{\prime}=0$ tells us that $g_{3}^{\prime}=0$ implies that $g_{3}=0$.

Subcase 3-3: $y_{m} \nmid y_{t}$ and $y_{t} \nmid y_{m}$. Since $\left(y_{t}, y_{m}\right)=y_{u}$, we have $n_{u} \neq 0$ by Lemma 2.3 (iii). So we also can find a $v \in A$ such that $y_{u}=\max \left(T_{l(v)}\right)$ as in Subcase 2-2. Then

where

$$
\sum_{s=1}^{n}\left(y_{m}, y_{s}\right)^{e} c_{s t}=\left[y_{m}, y_{t}\right]^{e}+y_{t}^{e} g_{4}
$$

$$
g_{4}=\sum_{s \in A \backslash\left\{t^{\prime}, m^{\prime}\right\}} \frac{\left(y_{s}, y_{m}\right)^{e}}{\left(y_{s}, y_{t}\right)^{e}}-\sum_{s \in A \backslash\left\{a_{1}, v\right\}} \frac{\left(T_{l(s)}, y_{m}\right)^{e}}{\left(T_{l(s)}, y_{t}\right)^{e}} .
$$

In what follows we show that $g_{4}=0$.

Subcase 3-3-1: $m^{\prime} \in A_{2}$. Since $y_{t} \nmid \max \left(T_{l(s)}\right)$ and $y_{t} \mid y_{t^{\prime}}$, by Lemma $2.3(\mathrm{i})$ we have $\left(T_{l(s)}, y_{t}\right)=\left(T_{l(s)}, y_{t^{\prime}}\right)$. Then

$$
\begin{aligned}
g_{4} & =h_{1}+\left(\sum_{s \in A_{2} \backslash\left\{m^{\prime}\right\}} \frac{\left(y_{s}, y_{m}\right)^{e}}{\left(y_{s}, y_{t}\right)^{e}}-\sum_{s \in A_{2} \cup\left\{t^{\prime}\right\} \backslash\left\{a_{1}, v\right\}} \frac{\left(T_{l(s)}, y_{m}\right)^{e}}{\left(T_{l(s)}, y_{t}\right)^{e}}\right) \\
& =h_{1}+\left(\sum_{s \in A_{2} \backslash\left\{m^{\prime}\right\}} \frac{\left(y_{s}, y_{m}\right)^{e}}{\left(y_{s}, y_{t^{\prime}}\right)^{e}}-\sum_{s \in A_{2} \cup\left\{t^{\prime}\right\} \backslash\left\{a_{1}, v\right\}} \frac{\left(T_{l(s)}, y_{m}\right)^{e}}{\left(T_{l(s)}, y_{t^{\prime}}\right)^{e}}\right)=: h_{1}+h_{10} .
\end{aligned}
$$

As in Subcase 2-2-1, we have $h_{1}=0$ since $t$ is independent of $t \in A$. Since $t^{\prime} \in A, h_{3}+h_{4}=0$ gives $h_{10}=0$. Therefore $g_{4}=0$.

Subcase 3-3-2: $m^{\prime} \in A_{1}$. Let $B$ be as in Subcase 2-2-2. Then

$$
g_{4}=h_{10}+h_{5}+\sum_{s \in A_{1} \backslash B}\left(\frac{\left(y_{s}, y_{m}\right)^{e}}{\left(y_{s}, y_{t}\right)^{e}}-\frac{\left(T_{l(s)}, y_{m}\right)^{e}}{\left(T_{l(s)}, y_{t}\right)^{e}}\right) \text {. }
$$

As in the proof of Subcase 2-2-2, we can show $g_{4}=0$. Part (i) is proved. 
(ii) By [31] we know that there is a gcd-closed set $S$ with $\max _{x \in S}\left\{\left|G_{S}(x)\right|\right\}$ $=2$ such that $\left(S^{e}\right)^{-1}\left[S^{e}\right] \notin M_{|S|}(\mathbb{Z})$. Now let $r \geq 3$ and $p_{1}<\cdots<p_{r}$ be prime numbers. Define $x_{1}=1, x_{i}=p_{i-1}(2 \leq i \leq r+1)$ and $x_{r+2}=p_{1} \cdots p_{r}$. Obviously $S:=\left\{x_{1}, \ldots, x_{r+2}\right\}$ is gcd-closed and $\max _{x \in S}\left\{\left|G_{S}(x)\right|\right\}=r$. By [4], we have

$$
\left(\left(S^{e}\right)^{-1}\right)_{i j}=\sum_{\substack{x_{i}\left|x_{k} \\ x_{j}\right| x_{k}}} \frac{c_{i k} c_{j k}}{b_{k}}
$$

where

$$
b_{i}=\sum_{\substack{d \mid x_{i} \\ d \nmid x_{t}, x_{t}<x_{i}}} J_{e}(d) \text { and } c_{i j}=\sum_{\substack{d x_{i} \mid x_{j} \\ d x_{i} \nmid x_{t}, x_{t}<x_{j}}} \mu(d) .
$$

From [19] we derive $b_{r+2}=\prod_{i=1}^{r} p_{i}^{e}-\sum_{i=1}^{r} p_{i}^{e}+r-1$. Using these and by some computations, we get $\left(\left(S^{e}\right)^{-1}\left[S^{e}\right]\right)_{22}=-\sigma / b_{r+2}$, where

$$
\sigma=\prod_{i=1}^{r} p_{i}^{e}-p_{1}^{e} \sum_{i=2}^{r} p_{i}^{e}+(r-2) p_{1}^{e} .
$$

Since $\sum_{i=2}^{r} p_{i}^{e}>r-1$, we have $b_{r+2}-\sigma>0$. Clearly $\sigma>0$. So $0<\sigma / b_{r+2}<1$ and $\left(\left(S^{e}\right)^{-1}\left[S^{e}\right]\right)_{22} \notin \mathbb{Z}$. Hence $\left(S^{e}\right) \nmid\left[S^{e}\right]$ as required. Part (ii) is proved.

REMARK. By Theorem 2.5(i) we know immediately that the sum of the elements of the $t$ th column of the matrix $C$ equals $y_{t}^{e}$. On the other hand, let $S_{0}$ be the gcd-closed set as in (1). Then by Theorem 2.5(i) we have $\left(S_{0}^{e}\right)^{-1}\left[S_{0}^{e}\right]=C$. For any general gcd-closed set $S$, let $\tau$ be the permutation such that $S_{\tau}=S_{0}$. It follows that $\left(S^{e}\right)^{-1}\left[S^{e}\right]=P^{t} C P$, where $P$ is the $n \times n$ permutation matrix formed by $\tau$. Now let $e \geq 1$ be a given integer and $S$ be a gcd-closed set with $\max _{x \in S}\left\{\left|G_{S}(x)\right|\right\} \geq 2$. It is of interest to have necessary and sufficient conditions on $S$ such that $\left(S^{e}\right) \mid\left[S^{e}\right]$ in $M_{|S|}(\mathbb{Z})$. This is an open problem.

3. The lcm-closed case. The reciprocal set of $S=\left\{x_{1}, \ldots, x_{n}\right\}$, denoted by $m S^{-1}$, is defined by $m S^{-1}:=\left\{m / x_{1}, \ldots, m / x_{n}\right\}$. By [19] we know that $m S^{-1}$ is gcd-closed if and only if $S$ is lcm-closed. We can now state the second main result of this paper.

THEOREM 3.1.

(i) Let $S$ be an lcm-closed set such that $\max _{x \in S}\left\{\left|L_{S}(x)\right|\right\}=1$ and $m S^{-1}$ be the same set as in (1). Then $\left(S^{e}\right)^{-1}\left[S^{e}\right]=\operatorname{diag}\left(x_{1}^{-e}, \ldots, x_{n}^{-e}\right) \cdot C$. $\operatorname{diag}\left(x_{1}^{e}, \ldots, x_{n}^{e}\right) \in M_{n}(\mathbb{Z})$. In particular, Conjecture 1.2 is true.

(ii) For each integer $r \geq 2$, there exists an lcm-closed set $S$ with $\max _{x \in S}\left\{\left|L_{S}(x)\right|\right\}=r$ such that the power $G C D$ matrix $\left(S^{e}\right)$ on $S$ does not divide the power $L C M$ matrix $\left[S^{e}\right]$ on $S$ in $M_{n}(\mathbb{Z})$. 
Proof. (i) Let $x_{i} y_{i}=m$ for all $1 \leq i \leq n$. Then $S=\left\{x_{1}, \ldots, x_{n}\right\}$ since $m S^{-1}=\left\{y_{1}, \ldots, y_{n}\right\}$. Since

$$
\left(x_{i}, x_{j}\right)=\frac{m}{\left[m / x_{i}, m / x_{j}\right]}=\frac{x_{i} x_{j}}{m} \cdot\left(\frac{m}{x_{i}}, \frac{m}{x_{j}}\right),
$$

we get

$$
\left(S^{e}\right)=1 / m^{e} \cdot D \cdot\left(\left(m S^{-1}\right)^{e}\right) \cdot D, \quad\left[S^{e}\right]=1 / m^{e} \cdot D \cdot\left[\left(m S^{-1}\right)^{e}\right] \cdot D,
$$

where $D=\operatorname{diag}\left(x_{1}^{e}, \ldots, x_{n}^{e}\right)$. We deduce that

$$
\left(S^{e}\right)^{-1}\left[S^{e}\right]=D^{-1}\left(\left(m S^{-1}\right)^{e}\right)^{-1}\left[\left(m S^{-1}\right)^{e}\right] D .
$$

Clearly it follows from $\max _{y \in S}\left\{\left|L_{S}(y)\right|\right\}=1$ that $\max _{y \in m S^{-1}}\left\{\left|G_{m S^{-1}}(y)\right|\right\}$ $=1$. So Theorem 2.5(i) applied to the set $m S^{-1}$ gives $\left(S^{e}\right)^{-1}\left[S^{e}\right]=D^{-1} C D$, where $C=\left(c_{i j}\right)$ is defined after Lemma 2.4. Hence the $(i, j)$ entry of the matrix $D^{-1} C D$ is

$$
\frac{x_{j}^{e}}{x_{i}^{e}} c_{i j}=\frac{x_{j}^{e}}{x_{i}^{e}} \frac{y_{j}^{e}}{\left(y_{i}, y_{j}\right)^{e}} \delta_{i j}=\frac{x_{j}^{e} y_{j}^{e}}{x_{i}^{e} y_{i}^{e}} \frac{y_{i}^{e}}{\left(y_{i}, y_{j}\right)^{e}} \delta_{i j}=\frac{y_{i}^{e}}{\left(y_{i}, y_{j}\right)^{e}} \delta_{i j} \in \mathbb{Z}
$$

since $\delta_{i j}$ is an integer. This implies that $\left(S^{e}\right)^{-1}\left[S^{e}\right] \in M_{n}(\mathbb{Z})$ as required.

(ii) It is known [31] that there is an lcm-closed set $S$ with $\max _{x \in S}\left\{\left|L_{S}(x)\right|\right\}$ $=2$ such that $\left(S^{e}\right)^{-1}\left[S^{e}\right] \notin M_{|S|}(\mathbb{Z})$. For $r \geq 3$, let $p_{1}<\cdots<p_{r}$ be primes and $m=p_{1} \cdots p_{r}$. Set $S=\left\{m, m / p_{1}, \ldots, m / p_{r}, 1\right\}$. Then $S$ is lcm-closed and $m S^{-1}=\left\{1, p_{1}, \ldots, p_{r}, p_{1} \cdots p_{r}\right\}$ is gcd-closed. By (2) and replacing $S$ by $m S^{-1}$ in the proof of Theorem 2.5 (ii), we get

$$
-1<\left(\left(S^{e}\right)^{-1}\left[S^{e}\right]\right)_{22}=\left(\left(\left(m S^{-1}\right)^{e}\right)^{-1}\left[\left(m S^{-1}\right)^{e}\right]\right)_{22}=-\frac{\sigma}{b_{r+2}}<0,
$$

where $\sigma$ and $b_{r+2}$ are as above. So $\left(S^{e}\right)^{-1}\left[S^{e}\right] \notin M_{r+2}(\mathbb{Z})$ as desired.

Remark. Given any integer $e \geq 1$. It is an open question to find necessary and sufficient conditions on an lcm-closed set $S$ with $\max _{x \in S}\left\{\left|L_{S}(x)\right|\right\}$ $\geq 2$ so that the power GCD matrix $\left(S^{e}\right)$ divides the power LCM matrix $\left[S^{e}\right]$ in $M_{|S|}(\mathbb{Z})$.

4. Remarks on the finite arithmetic progression case. The renowned Dirichlet theorem states that the arithmetic progression contains infinitely many primes if the first term and the common difference are coprime, while the Green-Tao theorem [11] says that the set of primes contains arbitrarily long arithmetic progressions. Farhi [9] and Hong-Feng [20] investigated the non-trivial lower bounds for the least common multiple of finite arithmetic progressions. Ligh [25] raised the problem of computing the determinants of Smith matrices on a finite arithmetic progression which is still open. We are interested in the divisibility of Smith matrices on a finite arithmetic progression. We can easily check that if $S=\{2,2+q, 2+2 q\}$ and $(2, q)=1$, then for all integer $e \geq 1$, we have $\left(S^{e}\right)^{-1}\left[S^{e}\right] \in M_{3}(\mathbb{Z})$. But the set 
$S=\{4,7,10\}$ gives $(S)^{-1}[S] \notin M_{3}(\mathbb{Z})$. Now fix an integer $e \geq 1$. We do not know how to characterize the arithmetic progression $S=\{a+b, \ldots, a+n b\}$, where $(a, b)=1$, such that $\left(S^{e}\right)^{-1}\left[S^{e}\right] \in M_{n}(\mathbb{Z})$. This problem remains open.

Acknowledgements. The authors would like to thank the anonymous referee for careful reading of the manuscript and valuable comments.

\section{References}

[1] T. M. Apostol, Arithmetical properties of generalized Ramanujan sums, Pacific J. Math. 41 (1972), 281-293.

[2] S. Beslin and S. Ligh, Another generalisation of Smith's determinant, Bull. Austral. Math. Soc. 40 (1989), 413-415.

[3] K. Bourque and S. Ligh, Matrices associated with classes of arithmetical functions, J. Number Theory 45 (1993), 367-376.

[4] - - - Matrices associated with arithmetical functions, Linear Multilinear Algebra 34 (1993), 261-267.

[5] - - -, Matrices associated with classes of multiplicative functions, Linear Algebra Appl. 216 (1995), 267-275.

[6] W. Cao, On Hong's conjecture for power LCM matrices, Czechoslovak Math. J. 57 (2007), 253-268.

[7] P. Codecá and M. Nair, Calculating a determinant associated with multiplicative functions, Boll. Un. Mat. Ital. Sez. B Artic. Ric. Mat. (8) 5 (2002), 545-555.

[8] L. E. Dickson, History of the Theory of Numbers, Vol. I, AMS Chelsea Publ., 1999.

[9] B. Farhi, Minorations non triviales du plus petit commun multiple de certaines suites finies d'entiers, C. R. Math. Acad. Sci. Paris 341 (2005), 469-474.

[10] W. Feng, Q. Tan and L. Zheng, A note on a conjecture of Hong of divisibility of LCM matrices, J. Sichuan Univ. Nat. Sci. Ed. 45 (2008), 41-42.

[11] B. Green and T. Tao, The primes contain arbitrarily long arithmetic progressions, Ann. of Math. (2) 167 (2008), 481-548.

[12] T. Hilberdink, Determinants of multiplicative Toeplitz matrices, Acta Arith. 125 (2006), 265-284.

[13] S. F. Hong, On the Bourque-Ligh conjecture of least common multiple matrices, J. Algebra 218 (1999), 216-228.

[14] -, Gcd-closed sets and determinants of matrices associated with arithmetical functions, Acta Arith. 101 (2002), 321-332.

[15] - On the factorization of LCM matrices on gcd-closed sets, Linear Algebra Appl. 345 (2002), 225-233.

[16] - Factorization of matrices associated with classes of arithmetical functions, Colloq. Math. 98 (2003), 113-123.

[17] —, Notes on power LCM matrices, Acta Arith. 111 (2004), 165-177.

[18] - Nonsingularity of least common multiple matrices on gcd-closed sets, J. Number Theory 113 (2005), 1-9.

[19] - Nonsingularity of matrices associated with classes of arithmetical functions on lcm-closed sets, Linear Algebra Appl. 416 (2006), 124-134.

[20] S. F. Hong and W. Feng, Lower bounds for the least common multiple of finite arithmetic progressions, C. R. Math. Acad. Sci. Paris 343 (2006), 695-698. 
[21] S. F. Hong and E. K. S. Lee, Asymptotic behavior of eigenvalues of reciprocal power LCM matrices, Glasgow Math. J. 50 (2008), 163-174.

[22] S. F. Hong and R. Loewy, Asymptotic behavior of eigenvalues of greatest common divisor matrices, ibid. 46 (2004), 551-569.

[23] S. F. Hong, K. P. Shum and Q. Sun, On nonsingular power LCM matrices, Algebra Colloq. 13 (2006), 689-704.

[24] M. Li, Notes on Hong's conjectures of real number power LCM matrices, J. Algebra 315 (2007), 654-664.

[25] S. Ligh, On Smith's determinant, Linear Multilinear Algebra 22 (1988), 305-306.

[26] P. Lindqvist and K. Seip, Note on some greatest common divisor matrices, Acta Arith. 84 (1998), 149-154.

[27] P. J. McCarthy, A generalization of Smith's determinant, Canad. Math. Bull. 29 (1986), 109-113.

[28] I. Niven and H. Zuckerman, An Introduction to the Theory of Numbers, 3rd ed., Wiley, New York, 1960.

[29] H. J. S. Smith, On the value of a certain arithmetical determinant, Proc. London Math. Soc. 7 (1875-1876), 208-212.

[30] A. Wintner, Diophantine approximations and Hilbert's space, Amer. J. Math. 66 (1944), 564-578.

[31] J. R. Zhao, S. F. Hong, Q. Liao and K. P. Shum, On the divisibility of power LCM matrices by power GCD matrices, Czechoslovak Math. J. 57 (2007), 115-125.

Mathematical College

Sichuan University

Chengdu 610064, P.R. China

E-mail: s-f.hong@tom.com hongsf02@yahoo.com jr-zhao@tom.com yinyouzhan78@163.com
J. R. Zhao's current address: School of Economic Mathematics Southwestern University of Finance and Economics Chengdu 610074, P.R. China

Received on 29.8.2007

and in revised form on 12.11.2007 